Research Article

\title{
Comparison of RPR and ELISA with TPHA for the Diagnosis of Syphilis: Implication for Updating Syphilis Point-of-Care Tests in Ethiopia
}

\author{
Markos Negash $(D$, Tadelo Wondmagegn, and Demeke Geremew \\ Department of Immunology and Molecular Biology, School of Biomedical and Laboratory Sciences, University of Gondar, \\ Gondar, Ethiopia
}

Correspondence should be addressed to Markos Negash; markosnegash@yahoo.com

Received 2 February 2018; Revised 24 April 2018; Accepted 27 May 2018; Published 8 July 2018

Academic Editor: David Smajs

Copyright ( 2018 Markos Negash et al. This is an open access article distributed under the Creative Commons Attribution License, which permits unrestricted use, distribution, and reproduction in any medium, provided the original work is properly cited.

\begin{abstract}
Background. Syphilis is a sexually transmitted disease (STD) caused by the spirochete Treponema pallidum, and it persists to be a major public health problem in Africa, including Ethiopia. Syphilis diagnosis is made by either nontreponemal or treponemal approaches, though in developing countries the diagnosis relies mostly on nonspecific tests due to several reasons. Thus, the objective of this study was to assess the sensitivity, specificity, predictive values, and agreement of rapid plasma reagin (RPR) and enzyme-linked immunosorbent assay (ELISA) with Treponema pallidum hemagglutination assay (TPHA) as a gold standard for the diagnosis of syphilis. Results. The sensitivity, specificity, and positive and negative predictive values of ECOTEST-RPR were $100 \%, 80.8 \%, 76.2 \%$, and $100 \%$, respectively. However, the sensitivity, specificity, and positive and negative predictive values of DIALAB-ELISA were $98.4 \%, 94.9 \%, 92.3 \%$, and $98.9 \%$, respectively. The agreement between DIALABELISA and Randox-TPHA was excellent (kappa value: 0.96 ) as compared to ECOTEST-RPR and Randox-TPHA assay (kappa value: 0.88 ). Conclusion. We found a characteristically variable performance of DIALAB-ELISA test and the currently available traditional ECOTEST-RPR test in the study area. The use of ECOTEST-RPR as a diagnostic test is confronted by its false positivity. Thus, neither the ECOTEST-RPR nor the DIALAB-ELISA test stands on its own to be used either as screening or confirmatory test for syphilis diagnosis. Consequently, thorough studies should be conducted aiming on a change of the current diagnostic scheme in the community.
\end{abstract}

\section{Background}

Syphilis is a sexually transmitted disease (STD) caused by the spirochete Treponema pallidum ( $T$. pallidum) which can be spread by sexual contact, by blood transfusion, and via vertical transmission [1]. Syphilis affects 12 million people annually and results in significant morbidity if not mortality. In sub-Saharan Africa, syphilis remains a serious public health problem. Prevalence of active syphilis infection among African countries showed $12.8 \%$ in Tanzania and Kenya [2,3]. But the magnitude of syphilis among blood donors in Gondar (Ethiopia) was $1.3 \%$ in 2010 [4].

Syphilis diagnosis can be made by several approaches. In addition to the mainstay serological diagnostic method, dark-field microscopy by which the spirochete is examined and observed from the lesion under dark-field microscope is also another method [5-7]. T. pallidum, the etiological agent of syphilis, produces at least two types of antibodies in human infections: treponemal antibodies that can be detected by fluorescent treponemal antibody absorption (FTA-ABS) and nontreponemal antibody (reagin) that can be detected by RPR antigen card or VDRL test. Nontreponemal tests such as the venereal disease research laboratory (VDRL) and rapid plasma reagin (RPR) are based on the reaction of cardiolipin with nonspecific antibodies produced in response to syphilitic infection [8]. However, this test lacks sensitivity and specificity due to several reasons including pregnancy, autoimmune disorders, infections, and stages of syphilis infection $[9,10]$. Therefore, treponemal-specific tests like enzyme immunoassay (EIA), 
T. pallidum hemagglutination assay (TPHA), microhemagglutination, fluorescent treponemal antibody absorption test (FTA-abs), and the enzyme-linked immunosorbent assay (ELISA) that detect IgG antibodies to antigenic components of T. pallidum are used primarily to confirm the diagnosis of syphilis in patients with a reactive nontreponemal test $[11,12]$. Despite the availability of relatively sensitive tests and affordable treatment, the disease remains a global health problem $[13,14]$.

Syphilis remains a major cause of reproductive morbidity and poor pregnancy outcomes in developing countries including Ethiopia. In $80 \%$ of infected pregnant women, it results in stillbirth and spontaneous abortion (40\%), perinatal death (20\%), and serious neonatal infections and lowbirth weight babies (20\%) [15-17]. Syphilis has also acquired a new potential for morbidity and mortality through an association with increased risk for HIV infection [18].

Screening of pregnant women, blood donors, and social workers (drivers and newly employed social workers) for syphilis is a routine activity at all healthcare institutions in Ethiopia. For this purpose, due to cost effectiveness, RPR which has questionable test performance is usually used as a screening tool in Ethiopia. However, despite reports of diagnostic performance provided by the manufacturers, data on test performance of RPR in the study area remain limited. Thus, the objective of this study was to assess the sensitivity, specificity, predictive values, and agreement of ECOTESTRPR and DIALAB-ELISA with Randox-TPHA assay as a gold standard for syphilis diagnosis among syphilis-suspected patients attending the University of Gondar Hospital (UGH), Northwest Ethiopia.

\section{Methods}

2.1. Study Design, Period, and Area. Facility-based crosssectional study was conducted in UGH, from November 2015 to June 2016. The University of Gondar Hospital is one of the pioneer teaching hospitals in Ethiopia, located in Amhara region, Northwest Ethiopia. The hospital has eight different laboratory sections, including Serology, which provides teaching, diagnostic, and research services for the university community, Gondar town inhabitants, and the nearby Woreda populations.

2.2. Study Participants. Following acquisition of informed consent, a total of 160 participants were included in this study. Out of them, 80 participants were diagnosed as positive for syphilis infection by the routinely used technique in the study area (ECOTEST-RPR) at the University of Gondar Teaching hospital Laboratory. Charts of all patients were reviewed to assess if specific therapy was commenced. Likewise, 80 apparently healthy individuals that do not have any history of sexually transmitted diseases were recruited from Gondar Blood Bank Center.

2.3. Specimen Collection and Processing. Blood sample was collected from each participant and centrifuged until the serum was separated, and sera were stored at $-20^{\circ} \mathrm{C}$ until the actual laboratory tests were performed. Before running
RPR, ELISA, and TPHA, the stored serum samples were thawed at $37^{\circ} \mathrm{C}$ in a water bath until the ice formed becomes completely dissolved. Thereafter, RPR, ELISA, and TPHA were done and results were achieved according to the kit's manufacturer's instructions. A specimen that shows an equivocal result for any of the tests was retested. This study was performed according to the Standards for Reporting Diagnostic Accuracy Studies (STARD) essential items for reporting diagnostic accuracy studies (http://www.equatornetwork.org/reporting-guidelines/stard).

2.3.1. ECOTEST-RPR. [Principle] The antigen used in the ECOTEST-RPR (Assure Tech, Hangzhou, China) kit is a modification of VDRL antigen, which contains microparticulate charcoal to enhance the visual difference between a positive and negative result. Patient sera mixed with a fine particle cardiolipin antigen which has been enhanced with cholesterol, lecithin, and charcoal will result in a macroscopically visible flocculation-type precipitation if the patient's serum contains reagin - an antibody formed against cardiolipin (The detailed procedure can be accessed from the manufacturer's instruction, Assure Tech, Hangzhou, China.) Interpretation for each test was done using controls (positive and negative) according to the manufacturer's instruction as reactive $(R)$, if clumping is seen, or nonreactive $(N R)$ - smooth suspension, no clumping, or slight roughness.

2.3.2. The DIALAB Syphilis IgG/IgM ELISA. [Principle] Using the antigen sandwich enzyme-linked method (ELISA), this syphilis IgG/IgM ELISA test (DIALAB, Germany) can detect anti-TP antibodies. Polystyrene microwell strips are precoated with recombinant Treponema pallidum antigens produced in E. coli. Recombinant TP antigens that are conjugated to horseradish peroxidase (HRP) conjugate are incubated in the microwells with the sample. The precoated antigens indicate the same epitopes as the HRP-conjugate antigens, but the hosts are different. If anti-TP is present in the sample during incubation, the conjugated and precoated antigens will be bound to the two-variable antibody domains, and what is captured on the solid phase is the specific antibody-antigen immunocomplex. It is important that chromogen solutions containing tetramethylbenzidine (TMB) and urea peroxide are added into the wells after the washing phase to remove sample and unbound conjugates. The colorless chromogens are hydrolyzed by the bound HRP conjugate to a blue-colored product when the antigen-antibodyantigen sandwich complex is present. At this point, the blue color turns yellow. This occurs after the reaction with sulfuric acid is stopped. What can be measured proportionally at this juncture is the amount of antibody in the sample with the amount of color. Colorless wells indicate negative anti-TP samples (The detailed procedure can be accessed from the manufacturer's instruction, DIALAB, Germany.)

(1) Results Interpretation. Each microplate should be considered separately when calculating and interpreting results of the assay, regardless of the number of plates concurrently processed. The results are calculated by relating each sample's optical density (OD) value to the cut-off value (CO) of the plate. If the cut-off reading is based on a single- 
filter plate reader, the results should be calculated by subtracting the blank well OD value from the print report values of samples and controls. In case the reading is based on a dual-filter plate reader, do not subtract the blank well OD from the print report values of samples and controls.

2.3.3. Randox TPHA. [Principle] TPHA (Randox Laboratories, UK) reagents are used to detect human serum antibody to $T$. pallidum by means of an indirect hemagglutination (IHA) method. Preserved avian erythrocytes are coated with antigenic components of pathogenic T. pallidum (Nichols strain). These test cells agglutinate in the presence of specific antibodies to T. pallidum and show characteristic patterns in microtitration plates. Any nonspecific reactions occurring are detected using the control cells, which are avian erythrocytes, not coated with T. pallidum antigens. Nonspecific reactions may also be absorbed out using these control cells. Antibodies to nonpathogenic treponemes are absorbed by an extract of Reiter's treponemes, included in the cell suspension. Test results are obtained in 45-60 minutes, and the cell agglutination patterns are both easily read and are longlasting. (The detailed procedure can be accessed from the manufacturer's instruction, Randox Laboratories, UK.)

(1) Interpretation of Results. When the test well is positive, the control well should be observed. The control cells should settle to a compact button. They should not be used as a comparison for nonreactive serum patterns since the control cells will give a more compact pattern than the test cells will. Agglutination in the control well indicates the presence of nonspecific agglutinins in the sample; the test should be reported as invalid. A serum that gives this result may be absorbed using the control cells as detailed under nonspecific absorption. A doubtful reaction with test cells should be reported as indeterminate.

2.4. Statistical Analysis. The data was cleaned and double entered on an Excel Spreadsheet and transported to SPSS version 20. JavaStat two-way contingency table analysis software (http://statpages.org/ctab2x2.html) was also used to calculate sensitivity, specificity, and predictive and kappa values. The test findings of ECOTEST-RPR and DIALAB-ELISA were compared with results of the reference method (Randox-TPHA). The kappa value was determined to evaluate the agreement between ECOTEST-RPR, DIALAB-ELISA, and Randox-TPHA.

\section{Results}

A total of 160 participants were involved in this study. 80 (50\%) of them were diagnosed with syphilis using RPR as a diagnostic test in the study area. However, 80 (50\%) of them were apparently healthy participants and were negative for syphilis by all types of tests (ECOTEST-RPR, DIALABELISA, and Randox-TPHA). The participants' age range was from 20 to 52 years old, and most of them (77\%) were between 22 to 32 years. Among the participants, 84 (52.5\%) of them were males and $76(47.5 \%)$ were females. Most of the study subjects $(107,66.9 \%)$ were from rural areas of the nearby inhabitants, and $53(33.1 \%)$ of the subjects were urban residents (Table 1). Among 40 patients who were diagnosed as having syphilis by the RPR test, 2 patients had primary syphilis, and 9 patients had secondary syphilis with clinical presentation of nonitchy maculopapular rash, condyloma lata, and generalized lymphadenopathy whereas clinical data of the rest RPR-positive patients were not fully documented on the medical charts.

The sensitivity, specificity, and predictive values of ECOTEST-RPR and DIALAB-ELISA in this study were evaluated by using Randox-TPHA as a gold standard for the diagnosis of syphilis. Thus, the sensitivity and specificity of ECOTEST-RPR for syphilis detection were 100 and 80.8\%, respectively. The positive predictive value (PPV) and negative predictive value (NPV) were 76.2 and $100 \%$, respectively. The agreement between Randox-TPHA and ECOTEST-RPR tests was good with a kappa value of 0.88 (Table 2).

Moreover, the sensitivity and specificity of DIALABELISA for syphilis detection were 98.4 and $94.9 \%$, respectively. The positive predictive value (PPV) and negative predictive value (NPV) were 92.3 and $98.9 \%$, respectively. The agreement between TPHA and ELISA tests was nearly perfect with a kappa value of 0.96 (Table 3).

In this study, we have revised the medical charts of each participant and found that all ECOTEST-RPR-positive patients were commenced appropriate medication. Moreover, we found two samples with equivocal test results for DIALAB-ELISA but reanalysis of these samples by DIALAB-ELISA and Randox-TPHA provides a positive result under both tests. Similarly, we reported 15 discrepant results (i.e., ECOTEST-RPR-positive but DIALAB-ELISAnegative) as a negative result following reanalysis and verified as negative by Randox-TPHA.

\section{Discussion}

Syphilis infection can be diagnosed using either the treponemal or the nontreponemal approach. Nucleic acid amplification techniques (NAAT) like polymerase chain reaction (PCR) have opened the way for the development of highly sensitive and specific point-of-care tests. Nevertheless, the use of NAAT methods in developing countries is limited due to their affordability and cost and the complexity of the techniques to be used by the existing human resources in diagnostic areas. Additionally, a definitive syphilitic diagnostic test based on the detection of Treponema-specific IgG has been made available. However, a nontreponemal technique like RPR is the most widely used including the study area, though it is unreliable as a positive result does not necessarily indicate treponemal infection. Thus, its application in screening blood donors, pregnant women, and social workers has been in question. Therefore, we assessed the performance of ECOTEST-RPR and DIALAB-ELISA assay with RandoxTPHA as a reference standard diagnostic test for syphilis.

In the current study, the overall sensitivity and specificity of ECOTEST-RPR as compared to Randox-TPHA were $100 \%$ and $80.8 \%$, respectively. In our study, the sensitivity and specificity of RPR were comparable to the findings reported from Portugal, Korea, and Nepal [19-21]. However, sensitivity alone was much higher compared to reports from 
TABLE 1: The detection of syphilis by Randox-TPHA reactivity among study participants at University of Gondar Hospital, $2015-2016$.

\begin{tabular}{|c|c|c|c|c|c|c|}
\hline Demography & & Total (\%) & Randox-TPHA Positive (\%) & Randox-TPHA Negative (\%) & COR $(95 \%$ CI $)$ & $\operatorname{AOR}(95 \% \mathrm{CI})$ \\
\hline \multirow[b]{2}{*}{ Gender } & Male & $84(52.5)$ & $33(39.3)$ & $51(60.7)$ & $1.11(0.58-2.10)$ & $1.15(0.59-2.25)$ \\
\hline & Female & $76(47.5)$ & $28(36.8)$ & $48(63.2)$ & 1 & $\begin{array}{c}1 \\
1111111\end{array}$ \\
\hline \multirow{3}{*}{ Age (years) } & $20-30$ & $121(75.6)$ & $48(39.7)$ & $73(60.3)$ & $0.76(0.35-1.66)$ & $0.75(0.34-1.67)$ \\
\hline & $31-40$ & $36(22.5)$ & $12(33.3)$ & $24(66.7)$ & 1 & 1 \\
\hline & $\geq 41$ & $3(1.9)$ & $1(33.3)$ & $2(66.7)$ & N/A N/N/A & N/A \\
\hline \multirow{2}{*}{ Residence } & Urban & $53(33.1)$ & $19(35.8)$ & $34(64.2)$ & $0.86(0.44-1.71)$ & $0.90(0.44-1.83)$ \\
\hline & Rural & $107(66.9)$ & $42(39.3)$ & $65(60.7$ & 1 & 1 \\
\hline
\end{tabular}

N/A: not considered during analysis; TPHA: T. pallidum hemagglutination assay; COR: crude odds ratio; AOR: adjusted odds ratio; CI: confidence interval; $\%$ : percent.

TABLE 2: Serology results of the ECOTEST-RPR and Randox-TPHA tests at the University of Gondar Hospital, 2015-2016.

\begin{tabular}{lccc}
\hline ECOTEST-RPR & Reactive & $\begin{array}{c}\text { Randox-TPHA } \\
\text { Nonreactive }\end{array}$ & Total \\
\hline Reactive & 61 & 19 & 80 \\
Nonreactive & 0 & 80 & 80 \\
Total & 61 & 99 & 160 \\
\hline
\end{tabular}

Sensitivity of ECOTEST-RPR (61 of 61 samples), 100\%; specificity of ECOTEST-RPR ( 80 of 99 samples), 80.8\%; agreement $(61+80=141$, of 160 samples), $88 \%$; positive predictive value (61/80) $76.2 \%$; and negative predictive value (80/80) $100 \%$.

TABle 3: Serology results of the DIALAB-ELISA and RandoxTPHA tests at the University of Gondar Hospital, 2015-2016.

\begin{tabular}{lccc}
\hline DIALAB-ELISA & Reactive & $\begin{array}{c}\text { Randox-TPHA } \\
\text { Nonreactive }\end{array}$ & Total \\
\hline Reactive & 60 & 5 & 65 \\
Nonreactive & 1 & 94 & 95 \\
Total & 61 & 99 & 160 \\
\hline
\end{tabular}

Sensitivity of DIALAB-ELISA (60 of 61 samples), 98.4\%; specificity of DIALAB-ELISA (94 of 99 samples), 94.9\%; agreement $(60+94=154$, of 160 samples), $96.25 \%$; positive predictive value (60/65) $92.3 \%$; and negative predictive value (94/95) $98.9 \%$.

several studies [22-26]. The highest (100\%) sensitivity in our finding could be as follows: First, our participants may not have only syphilis infection, suggesting cross-reactivity of ECOTEST-RPR with cholesterol, lecithin, and cardiolipin antigens found in other disease processes as a result of cellular destruction. Second, it may be due to variation in protocols from different companies as well; in fact, it should not be forgotten that the diagnostic performance of RPR is profoundly affected by the stages of syphilis infection, which is not fully addressed in our study. Based on our finding, the highest sensitivity (100\%) is considered as a limitation of the ECOTEST-RPR serological test as its false positivity is enormous due to cross-reactive antibodies. Also, the chance of missing syphilitic cases at different stages is there because of the prozone effect. This high sensitivity of RPR has a negative implication in that wrongly diagnosed individuals are supposed to take medication together with their sexual partners (if there); this phenomenon results in economical, drug resistance, and social impacts in the community. Besides, blood from donors that are incorrectly diagnosed and labeled as positive will be discarded. Therefore, in the presence of this limitation and with the availability of other syphilis screening tests, the sole utilization of ECOTEST$\mathrm{RPR}$ assay routinely as a diagnostic method remains of great concern in the study area.

Nevertheless, the specificity of the test in our study showed lowest performances compared to the Indian and South African report (96.96\% and $100 \%$, resp.) $[22,27]$ and incomparably very high with respect to findings from Turkey and Latvia (0\%) $[23,28]$. In contrast to findings from studies, those come up with a superior specificity of RPR [20,27, 28], and we found a low specificity of manual ECOTEST-RPR test performance. The likely explanation may be the variation in the type of methods between our study and theirs. Although $\mathrm{RPR}$ is generally a nontreponemal test, we used a conventional manual ECOTEST-RPR test method while their method was the automated RPR test, indicating that the automated one has reduced interpersonal differences and other confounders. Furthermore, the clinical stage of study participants (primary, secondary, and tertiary syphilis) affects the specificity of the test because of the prozone phenomenon. Moreover, since reporting of the RPR test result (as positive or negative) is based on observation, the subjective (interpersonal) variation and subsequent decisions between laboratory analysts potentially affect the specificity of the test result.

Following the innovative introduction of ELISA and recombinant DNA (rDNA) technology for the diagnosis of syphilis, the traditional two-step approach of first screening with a nontreponemal test and then using a Treponema-specific confirmation test has been confronted and, as result, two diagnostic thoughts appeared. One school promotes the use of EIA as a screening test which needed to be confirmed by another test (technology or method but with equal or higher specificity), and the other school proposes the traditional approach of an algorithm [29].

Accepting either the traditional or the other approach, evaluating the performance of ELISA with reference to a better method is compulsory. In contrast to the ECOTEST-RPR test, DIALAB-ELISA has come up with a better test performance with reference to Randox-TPHA. In this study, we found 5 false positives with the DIALAB-ELISA test, thus 
giving a false-positive diagnostic rate of $8 \%$; this appears to be the least value as compared to that of RPR (31\%).

The sensitivity and specificity of DIALAB-ELISA with respect to Randox-TPHA are $98.4 \%$ and $94.9 \%$, respectively. Findings obtained from several studies have come up with a sensitivity and specificity ranging from 90 to $100 \%$, by far a result that is aligned with our study [23,28-32]. Despite these similarities, it should not be forgotten that other findings [33-35] on the performance of ELISA moderately differ from the results of the current study. The most probable reason for this performance variation may be due to the type of immunodominant syphilis proteins that are incorporated into the wells of ELISA kits.

During the evaluation of comparative prediction of a diagnostic kit with a reference standard, several issues will influence the interpretation of their results of which the prevalence/magnitude of a disease is the most important factor [36].

In the current study, the positive and negative predictive values of ECOTEST-RPR are 76.2 and 100\%, respectively. In the presence of variability on study participants (considering $50 \%$ of participants were positive for ECOTEST-RPR in this study), several studies come up with predictive values of ECOTEST-RPR similar with those of the current study [19, $21,26]$ whereas different prediction values of ECOTESTRPR have been reported from other studies [23]. The PPV and NPV of DIALAB-ELISA were $92.3 \%$ and $98.9 \%$, respectively, in this study. Likewise, reports from studies showed PPVs and NPVs which are comparable with ours [23, 29, $34]$, whereas variable predictive values were seen from a Turkish study [23]. As we have stated earlier, the predictive values in this study (for ECOTEST-RPR and DIALABELISA) were comparable and variable while comparing with PVs from several studies; a crucial point is that prevalence affects the PVs of any test. This implies that the same diagnostic test will have a different predictive accuracy according to the clinical setting/nature of study participants; thus, we strongly enlighten that the recommendations made in this study are based on the $50 \%$ magnitude of syphilis and that other studies should recognize the influence of the prevalence of disease when considering predictive values of diagnostic or screening tests.

The reported test performance of DIALAB-ELISA (sensitivity, specificity, and predictive values) in this study is encouraging and makes the test a better choice of the diagnosis approach in syphilis-suspected cases. Moreover, the agreement between DIALAB-ELISA and TPHA was nearly perfect (kappa value 0.96) as compared to that of ECOTEST-RPR and Randox-TPHA (kappa value of 0.88). Besides the superior performance, the ELISA technique has plenty of advantages over conventional flocculation screening tests (RPR). The method is designed potentially to be automated plus the reading of results is usually carried out by a microtiter plate reader, thus making the interpretation of results objective, unlike that of the RPR test which is subjective and hence requires having extensive experience. Unlike RPR, concerns like the prozone phenomenon and stage of syphilis infection do not affect the ELISA method. Regardless of all the above rational benefits, we want to enlighten the readers not to underestimate the drawbacks of ELISA methods.

\section{Conclusion}

This study comes up with a characteristically variable diagnostic performance of the DIALAB-ELISA test and the currently available traditional ECOTEST-RPR test in Ethiopia as compared to Randox-TPHA. ELISA kits with the recombinant T. pallidum antigens have certain attractions as a diagnostic tool. However, we cautioned the efficacy of the independent application of both ECOTEST-RPR and ELISA as a screening/diagnostic test for syphilis infection. In addition, it is important to underline that healthcare providers must perform a thorough review of each patient's clinical and treatment history while choosing the type of test and interpreting the results of RPR and ELISA IgG/IgM tests for syphilis diagnosis. Consequently, thorough studies should be conducted, aiming for a change of the current diagnostic scheme used in the community.

$\begin{array}{ll}\text { Abbreviations } \\ \text { CIs: } & \text { Confidence intervals } \\ \text { EIA: } & \text { Enzyme immunoassay } \\ \text { ELISA: } & \text { Enzyme-linked immunosorbent assay } \\ \text { FTA-abs: } & \text { Fluorescent treponemal antibody absorption test } \\ \text { IgG: } & \text { Immunoglobulin G } \\ \text { IRB: } & \text { Institutional Review Board } \\ \text { NPV: } & \text { Negative predictive value } \\ \text { NAAT: } & \text { Nucleic acid amplification techniques } \\ \text { PCR: } & \text { Polymerase chain reaction } \\ \text { PPV: } & \text { Positive predictive value } \\ \text { RPR: } & \text { Rapid plasma reagin } \\ \text { rDNA: } & \text { Recombinant DNA } \\ \text { STARD: } & \text { Standards for Reporting Diagnostic Accuracy } \\ & \text { Studies } \\ \text { STD: } & \text { Sexually transmitted disease } \\ \text { TPHA: } & \text { T. pallidum hemagglutination } \\ \text { T pallidum: } & \text { Treponema pallidum } \\ \text { UGH: } & \text { University of Gondar Hospital } \\ \text { VDRL: } & \text { Venereal disease research laboratory. }\end{array}$

\section{Data Availability}

The authors confirm that all data supporting our findings are contained within the manuscript and are fully available without restriction.

\section{Ethical Approval}

Ethical clearance was obtained from the University of Gondar Research Ethics Committee. Participation was voluntary, and informed consent was obtained from all subjects who accepted to participate in the study. Participants' willingness was asked verbally after briefly explaining the objectives of the study, the risks and benefits of the procedures, and the right not to participate in the study using their local language. The authors received verbally informed consent 
before including any of the participants in this study. Written consent was not acquired because syphilis-positive participants were recruited from the outpatient department laboratory of the Gondar University Hospital where they were sent to undergo a syphilis antibody test. Similarly, 80 apparently healthy participants with no history of sexually transmitted diseases were recruited from the hospital blood bank; thus, in both groups, we did not took any additional specimen but rather used the already provided blood sample that they provide at the hospital laboratory and blood bank. The additional sociodemographic data collection was a noninvasive procedure with no risk associated to it. Therefore, considering all these facts, only verbal agreement was acquired to be included in the study. Thereafter, only voluntary participants who are willing to give us a verbal consent (agree to participate) were recruited into the study.

\section{Conflicts of Interest}

The authors declare that they have no competing interest with regard to the present study.

\section{Authors' Contributions}

Markos Negash and Demeke Geremew conceived the study concept and designed the study; Markos Negash and Tadelo Wondmagegn carried out the data collection and laboratory analysis; Demeke Geremew and Tadelo Wondmagegn supervised the data collection and laboratory analysis; Markos Negash, Demeke Geremew, and Tadelo Wondmagegn analyzed the data and prepared the first manuscript draft; and Markos Negash and Demeke Geremew reviewed the draft. All authors read and approved the final manuscript.

\section{Acknowledgments}

The authors would like to acknowledge their technical assistant, Mister Amare Kifle, for his excellent technical support during the conduct of the study. The authors' gratitude also goes to all participants in the study and the University of Gondar Hospital. The authors are also thankful to the Gondar Blood Bank Center staff for their unreserved support during the study.

\section{References}

[1] P. Murray, K. Rosenthal, G. Kobayashi, and M. Pfaller, Medical Microbiology, Mosby company St. Loius, 4th edition, 2002.

[2] J. Todd, K. Munguti, H. Grosskurth et al., "Risk factors for active syphilis and TPHA seroconversion in a rural African population," Sexually Transmitted Infections, vol. 77, no. 1, pp. 37-45, 2001.

[3] M. Temmerman, K. Fonck, F. Bashir et al., "Declining syphilis prevalence in pregnant women in Nairobi since 1995: another success story in the STD field?," International Journal of STD \& AIDS, vol. 10, no. 6, pp. 405-408, 1999.

[4] B. Tessema, G. Yismaw, A. Kassu et al., "Seroprevalence of HIV, HBV, HCV and syphilis infections among blood donors at Gondar University Teaching Hospital, Northwest Ethiopia: declining trends over a period of five years," BMC Infectious Diseases, vol. 10, no. 1, 2010.

[5] S. A. Lasren, B. M. Steiner, and A. H. Rudolph, "Laboratory diagnosis and interpretation of tests for syphilis," Clinical Microbiology Reviews, vol. 8, no. 1, pp. 1-21, 1995.

[6] Syphilis, January 2003, from http://www.niaid.nih.gov/ factsheets/stdsyph.htm.

[7] E. C. Tramont, "Treponema pallidum (syphilis)," in Mandell, Douglas, and Bennett's Principles and Practice of Infectious Diseases, G. L. Mandell, J. E. Bennett, and R. Dolin, Eds., pp. 24742490, Churchill Livingstone, Philadelphia, 5th edition, 2000.

[8] A. F. Luger, "Serological diagnosis of syphilis: current methods," in Immunological Diagnosis of Sexually Transmitted Diseases, H. Young and A. McMillan, Eds., pp. 250-259, Dekker, New York, 1988.

[9] F. T. Fischbach, "Syphilis detection tests," in A Manual of Laboratory \& Diagnostic Tests, pp. 581-583, Lippincott, Philadelphia, 6th edition, 2000.

[10] M. C. Cummings, S. A. Lukehart, C. Marra et al., "Comparison of methods for the detection of Treponema pallidum in lesions of early syphilis," Sexually Transmitted Diseases, vol. 23, no. 5, pp. 366-369, 1996

[11] H. Young, A. Moyes, L. Seagar, and A. McMillan, "Novel recombinant-antigen enzyme immunoassay for serological diagnosis of syphilis," Journal of Clinical Microbiology, vol. 36, no. 4, pp. 913-917, 1998.

[12] B. Carlsson, H. S. Hanson, J. Wasserman, and A. Brauner, "Evaluation of the fluorescent treponemal antibody-absorption (FTA-Abs) test specificity," Acta Dermato-Venereologica, vol. 71, no. 4, pp. 306-311, 1991.

[13] World Health Organization, Prevalence and incidence of selected sexually transmitted infections, Chlamydia trachomatis, Neisseria gonorrhoeae, syphilis, and Trichomonas vaginalis: methods and results used by WHO to generate 2005 estimates, WHO, Geneva, Switzerland, 2011.

[14] R. W. Peeling and E. W. Hook, "The pathogenesis of syphilis: the great mimicker, revisited," The Journal of Pathology, vol. 208, no. 2, pp. 224-232, 2006.

[15] World Health Organization, "Action for the global elimination of congenital syphilis: rationale and strategy," in WHO Department of Reproductive Health and Research, WHO, Geneva, 2005.

[16] World Health Organization, "Detecting sexually transmitted infections: reproductive tract infections," http://www.who. int/reproductivehealth/publications/rtis/9241592656/en/.

[17] World Health Organization, "Sexually transmitted infections: reproductive tract infection assessment in pregnancy, childbirth and the postpartum period," January 2008, http://www. nacp.go.tz/site/download/stitrainerguideline.

[18] A. B. Olokoba, L. B. Olokoba, F. K. Salawu, A. Danburam, O. O. Desalu, J. K. Midala et al., "Syphilis HIV co-infection in northeastern Nigeria," International Journal of Tropical Medicine, vol. 3, no. 3, pp. 70-72, 2008.

[19] S. P. Dumre, G. Shakya, D. Acharya, S. Malla, and N. Adhikari, "Diagnostic dilemma of the single screening test used in the diagnosis of syphilis in Nepal," Nepal Medical College Journal, vol. 13, no. 4, pp. 238-240, 2011.

[20] J.-H. Lee, C. S. Lim, M.-G. Lee, and H.-S. Kim, "Comparison of an automated rapid plasma reagin (RPR) test with the conventional RPR card test in syphilis testing," BMJ Open, vol. 4, no. 12, article e005664, 2014. 
[21] A. Rakotoarisoa, H. Andriamandimbisoa, T. Randriamahazo, J. Andrianavalona, D. Rajaonatahiana, and A. Rasamindrakotroka, "Performance of SD Bioline Syphilis 3.0 for the Diagnosis of Sypilis a UPFR in Immunology of CHU-JRA," International Journal of Current Microbiology and Applied Sciences, vol. 6, no. 8, pp. 783-788, 2017.

[22] M. Paul and S. S. Sen, "A comparative study of clinically suspected syphilis cases with serological test VDRL/RPR \& Treponema pallidum hemagglutination assay (TPHA) in a tertiary care hospital (Silchar Medical College \& Hospital)," Journal of Science, vol. 6, no. 12, pp. 521-526, 2016.

[23] Y. Saral, A. R. Dilek, N. Dilek, İ. Bahçeci, and D. Z. Ulusan, "Serologic diagnosis of syphilis: comparison of different diagnostic methods," Acta Dermatovenerologica Croatica, vol. 20, no. 2, pp. 84-88, 2012.

[24] B. West, G. Walraven, L. Morison, J. Brouwers, and R. Bailey, "Performance of the rapid plasma reagin and the rapid syphilis screening tests in the diagnosis of syphilis in field conditions in rural Africa," Sexually Transmitted Infections, vol. 78, no. 4, pp. 282-285, 2002.

[25] P. J. Montoya, S. A. Lukehart, P. E. Brentlinger et al., "Comparsion of the diagnostic accuracy of a rapid immunochromatographic test and the rapid plasma reagin test for antenatal syphilis screening in Mozambique," Bulletin of the World Health Organization, vol. 84, no. 2, pp. 97-104, 2006.

[26] F. Terris-Prestholt, P. Vickerman, S. Torres-Rueda et al., "The cost-effectiveness of 10 antenatal syphilis screening and treatment approaches in Peru, Tanzania, and Zambia," International Journal of Gynecology \& Obstetrics, vol. 130, pp. S73-S80, 2015.

[27] M. P. Henning, C. Krüger, and L. Fletcher, "Syphilis seropositivity in recently admitted and long-term psychiatric inpatients: screening, prevalence and diagnostic profile," South African Journal of Psychiatry, vol. 18, no. 4, p. 5, 2012.

[28] D. Ozolins, S. Katkovska, L. Bobojeda, and A. Rancane, "Screening assays to find out late latent syphilis cases-which is the best one?," Internet Journal of Medical Update - Ejournal, vol. 4 , no. 2, 2009.

[29] V. Woznicová and Z. Vališová, "Performance of CAPTIA SelectSyph-G enzyme-linked immunosorbent assay in syphilis testing of a high-risk population: analysis of discordant results," Journal of Clinical Microbiology, vol. 45, no. 6, pp. 1794-1797, 2007.

[30] A. Ebel, L. Vanneste, M. Cardinaels et al., "Validation of the INNO-LIA syphilis kit as a confirmatory assay for Treponema pallidum antibodies," Journal of Clinical Microbiology, vol. 38, no. 1, pp. 215-219, 2000.

[31] A. Ebel, L. Bachelart, and J. M. Alonso, "Evaluation of a new competitive immunoassay (BioElisa Syphilis) for screening for Treponema pallidum antibodies at various stages of syphilis," Journal of Clinical Microbiology, vol. 36, no. 2, pp. 358361, 1998

[32] M. Zrein, I. Maure, F. Boursier, and L. Soufflet, "Recombinant antigen-based enzyme immunoassay for screening of Treponema pallidum antibodies in blood bank routine," Journal of Clinical Microbiology, vol. 33, no. 3, pp. 525-527, 1995.

[33] R. S. W. Tsang, I. E. Martin, A. Lau, and P. Sawatzky, "Serological diagnosis of syphilis: comparison of the Trep-Chek IgG enzyme immunoassay with other screening and confirmatory tests," FEMS Immunology \& Medical Microbiology, vol. 51, no. 1, pp. 118-124, 2007.
[34] I. Rodriguez, E. L. Alvarez, C. Fernandez, and A. Miranda, "Comparison of a recombinant-antigen enzyme immunoassay with Treponema pallidum hemagglutination test for serological confirmation of syphilis," Memórias do Instituto Oswaldo Cruz, vol. 97, no. 3, pp. 347-349, 2002.

[35] N. K. Naidu, Z. S. Bharucha, V. Sonawane, and I. Ahmed, "Comparative study of treponemal and non-treponemal test for screening of blood donated at a blood center," Asian Journal of Transfusion Science, vol. 6, no. 1, pp. 32-35, 2012.

[36] K. J. van Stralen, V. S. Stel, J. B. Reitsma, F. W. Dekker, C. Zoccali, and K. J. Jager, "Diagnostic methods I: sensitivity, specificity, and other measures of accuracy," Kidney International, vol. 75, no. 12, pp. 1257-1263, 2009. 


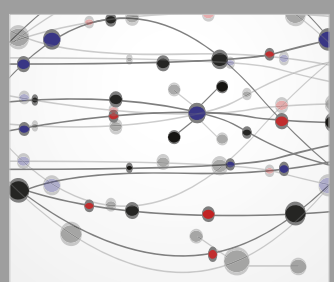

The Scientific World Journal
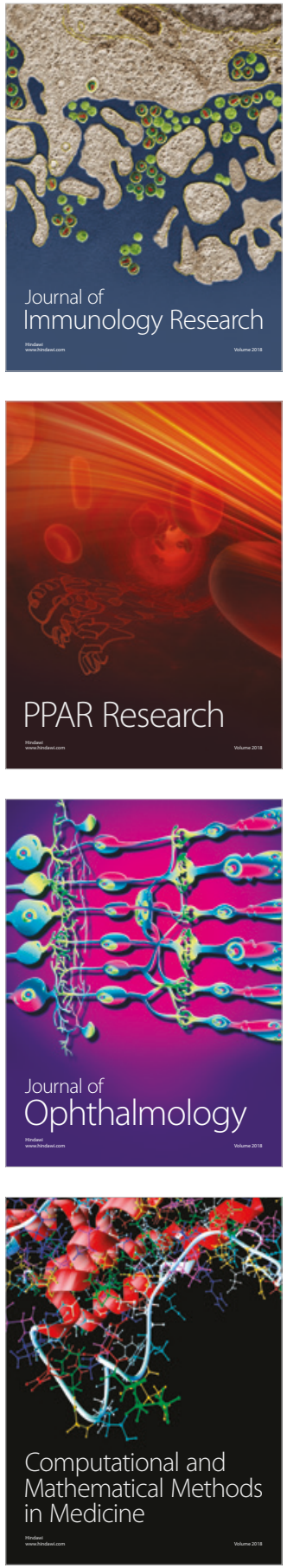

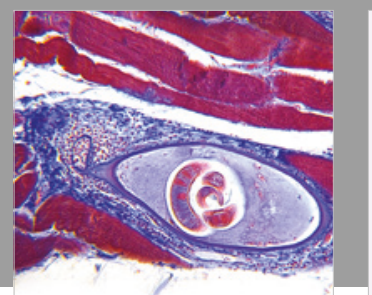

Gastroenterology Research and Practice

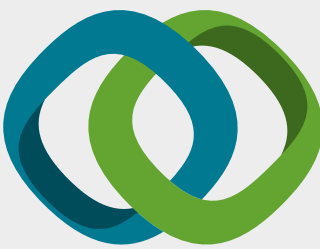

\section{Hindawi}

Submit your manuscripts at

www.hindawi.com
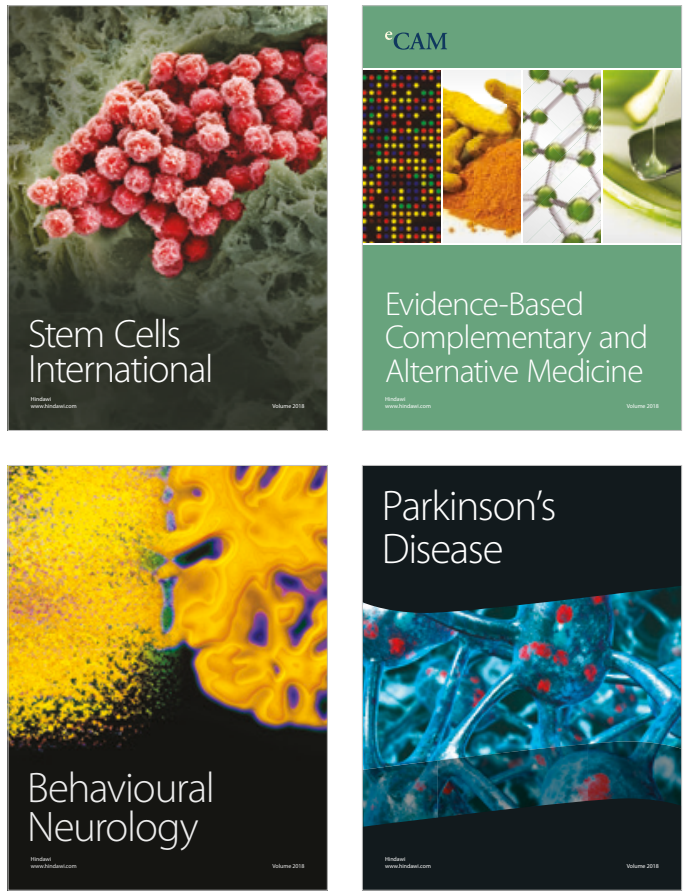

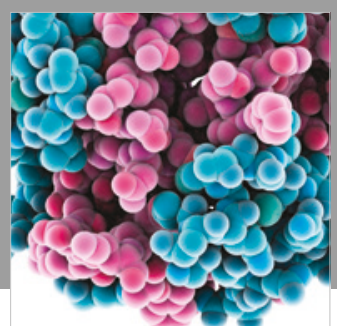

ournal of

Diabetes Research

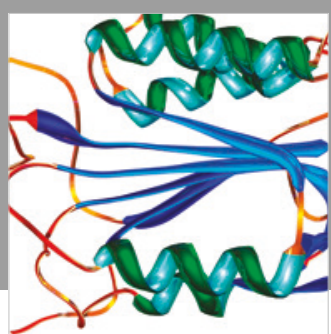

Disease Markers
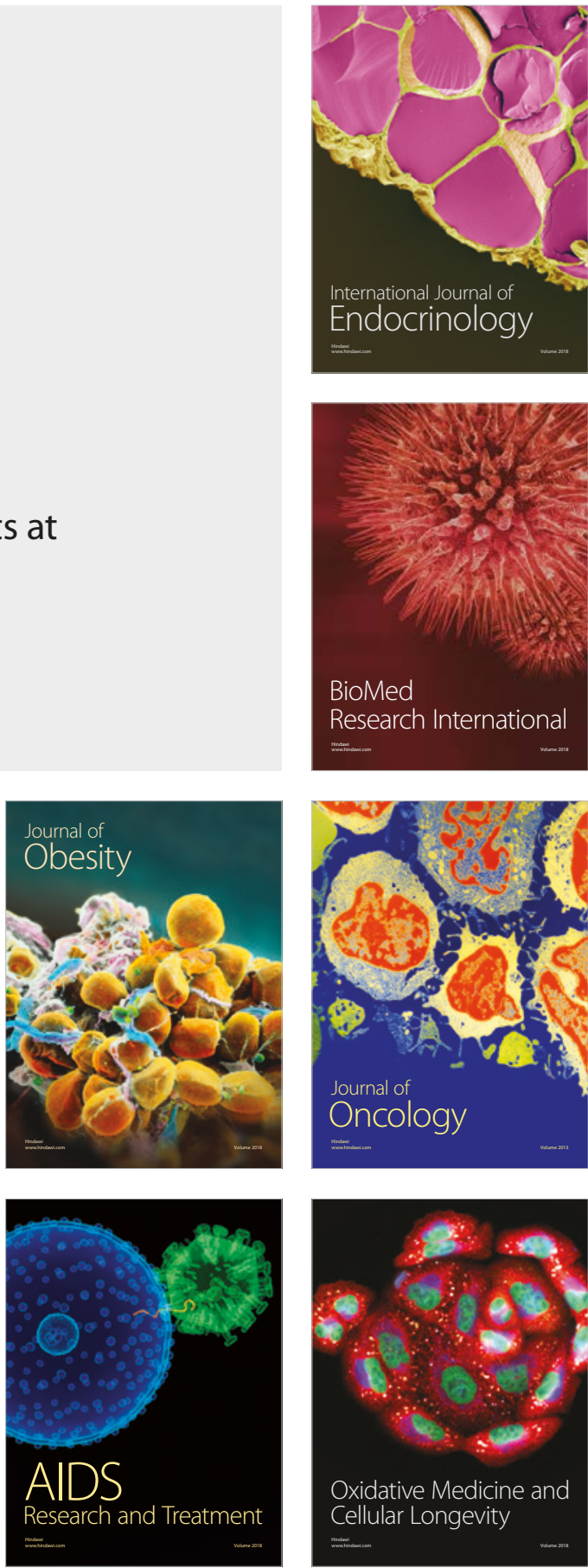